\title{
MITO, a new directional muon telescope design. First observations
}

\section{S. Ayuso ${ }^{1}$}

Universidad de Alcalá

Plaza de S. Diego s/n, Alcalá de Henares, Spain

E-mail: sindulfo. ayusoledu. uah.es

\section{J. I. García Tejedor}

Universidad de Alcalá

Plaza de S. Diego s/n, Alcalá de Henares, Spain

E-mail: ignacio.garcia@uah.es

\section{J.J. Blanco ${ }^{2}$}

Universidad de Alcalá

Plaza de S. Diego s/n, Alcalá de Henares, Spain

E-mail: juanjo.blanco@uah.es

\section{R. Gómez-Herrero}

Universidad de Alcalá

Plaza de S. Diego s/n, Alcalá de Henares, Spain

E-mail: raul.gomezh@uah.es

\section{O. García Población}

Universidad de Alcalá

Plaza de S. Diego s/n, Alcalá de Henares, Spain

E-mail: oscar.gpoblacioneuah.es

\section{J. Medina}

Universidad de Alcalá

Plaza de S. Diego s/n, Alcalá de Henares, Spain

E-mail: jose.medina@calmanm.es

\section{Prieto}

Universidad de Alcalá

Plaza de S. Diego s/n, Alcalá de Henares, Spain

E-mail: manuel.prieto@uah.es

\section{A. López-Comazzi}

Universidad de Alcalá

Plaza de S. Diego s/n, Alcalá de Henares, Spain

E-mail: alejandrofrancisco.l@edu.uah.es

\footnotetext{
${ }^{1}$ This work has been supported by the project CTM2016-77325-C2-1-P funded by Ministerio de

${ }^{2}$ Speaker

(C) Copyright owned by the author(s) under the terms of the Creative Commons

Attribution-NonCommercial-NoDerivatives 4.0 International License (CC BY-NC-ND 4.0).
} 
The Muon Impact Tracer and Observer (MITO) is a new cosmic ray detector, part of the Antarctic Cosmic Ray Observatory (ORCA), recently deployed by the University of Alcalá at the Juan Carlos I Spanish Antarctic Station (JCI) on Livingston Island, Antarctic Peninsula (S62 39'46", W60²3'20”, $12 \mathrm{~m}$ asl). MITO is composed of two stacked $100 \mathrm{~cm}$ x $100 \mathrm{~cm}$ x 5 cm BC-400 organic scintillators, eight photomultiplier tubes (PMTs) with corresponding light guides, a $16 \mathrm{~cm}$ lead separation layer, and an electronic data acquisition module able to register muon flux and pulse amplitude data. The goal of this setup is to provide a real-time estimation of the muon impact points on each scintillator and, thus, the muon arrival directions, based on the comparison of the pulse heights registered by each PMT. The electronic data acquisition module has been designed using different approaches and processing technologies (analogue and digital) producing some theoretically equivalent and redundant results which can be compared and used for better reliability. In this work we present the MITO design and discuss the preliminary results of the latitudinal survey of cosmic ray fluxes performed during its cruise aboard the Sarmiento de Gamboa oceanographic research vessel from Vigo (Spain) to the Antarctic Peninsula, from November $14^{\text {th }}, 2018$ to January $2^{\text {nd }}, 2019$.

36th International Cosmic Ray Conference -ICRC2019-

July 24th - August 1st, 2019

Madison, WI, U.S.A. 


\section{Introduction}

Cosmic rays (CRs) are energetic particles that constantly rain through the atmosphere. At sea level, relativistic muons and neutrinos are the most abundant secondary particles originated by the interactions of primary cosmic rays at the top of the atmosphere and they are able to penetrate deeply underground [1]. CRs are modulated by solar disturbances like Coronal Mass Ejections (CMEs) and shock waves even before they reach the Earth, thus magnetic clouds (MC) and shocks could be forecasted several hours in advance by ground based cosmic ray detectors like neutron monitors and muon telescopes. CMEs and shock waves are detectable with multidirectional muon telescopes earlier than with neutron monitors because muon detectors respond to higher energy cosmic rays (greater than $50 \mathrm{GeV}$ ) than neutron monitors (about $10 \mathrm{GeV}$ ) [2]. However, multidirectional muon telescopes as those of Nagoya (Japan), are not easily affordable because of their size and cost [3].

In this work, we present the MITO design concept. The design uses only two scintillators and eight PMTs, and it has been conceived with the goal of achieving not only muon flux registering, but also muon arrival directions through the capture and analysis of multiple PMT pulse amplitude data. The first prototype has traveled from Spain to Antarctica (where it is now installed) aboard the Sarmiento de Gamboa oceanographic vessel recording data. Here we are presenting the preliminary results.

\section{MITO design and implementation}

The telescope is composed of two identical devices, one stacked above the other $136.5 \mathrm{~cm}$ apart, each consisting of a BC-400 organic scintillator $(100 \mathrm{~cm} \times 100 \mathrm{~cm} \times 5 \mathrm{~cm}$, poly-vinyltoluene with $65 \%$ anthracene) and four PMTs laid out around the four sides of said scintillator, pointing inwards and at a $50 \mathrm{~cm}$ distance of the side (figure 1).

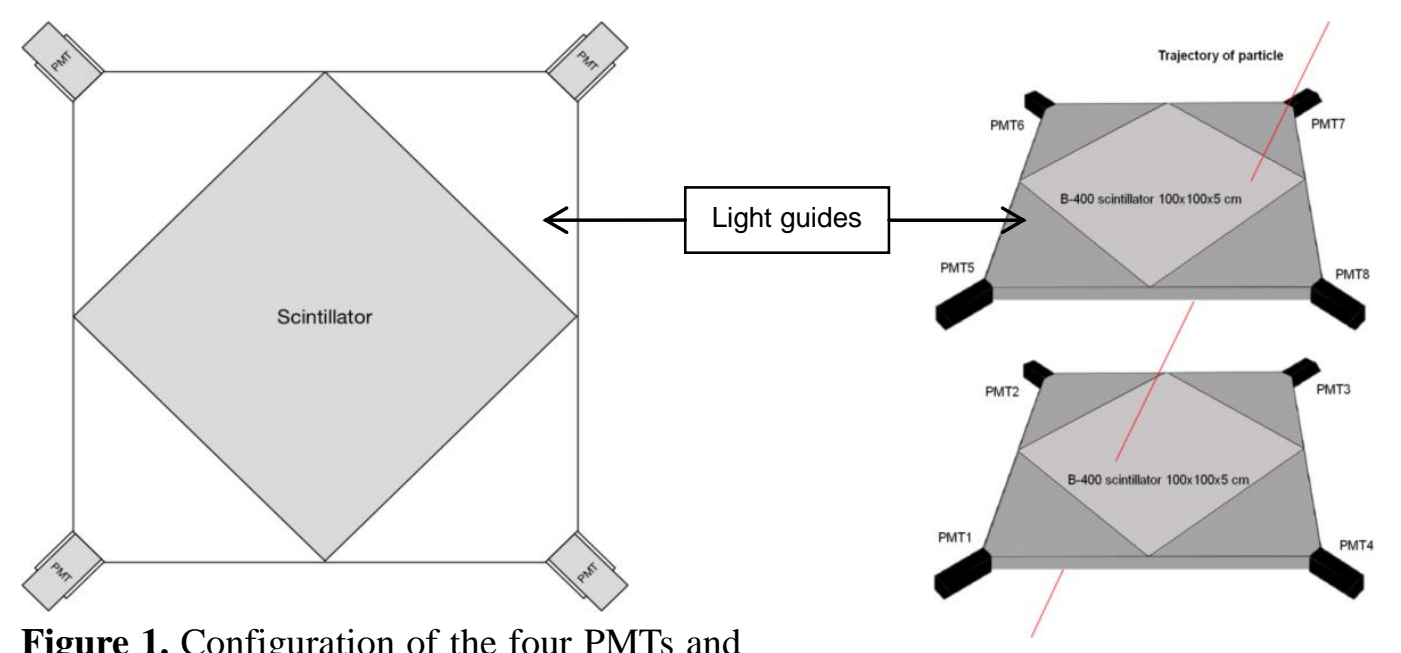

Figure 1. Configuration of the four PMTs and pyramidal light guides to determine the particle trajectory in the BC-400 scintillator.

Figure 2. Layout of the detection devices to determine muon trajectory.

PMTs have been consecutively numbered from one to eight proceeding clockwise around the scintillators, starting from one lateral side of the bottom scintillator (PMT1 to PMT4) and continuing (PMT5 to PMT8) at the same lateral side of the top scintillator (Figure 2). The 
device is built using a white-painted aluminum enclosure, so that the four PMTs are coupled to the corresponding scintillator by means of an aluminium pyramidal light guide. Each PMT collects the light coming out of its corresponding lateral surface, subsequently generating a pulse whose amplitude carries information both about the deposited energy and the distance between the particle trajectory in the scintillator and the corresponding lateral surface of the BC400 nearest to the PMT. Thus, the location of the impact trajectory can be calculated at each device by comparing the signal level of the pulses in coincidence and, by using two of these devices working in coincidence as in the presented stacked configuration, the two impact points determine the muon incident direction (Figure 2).

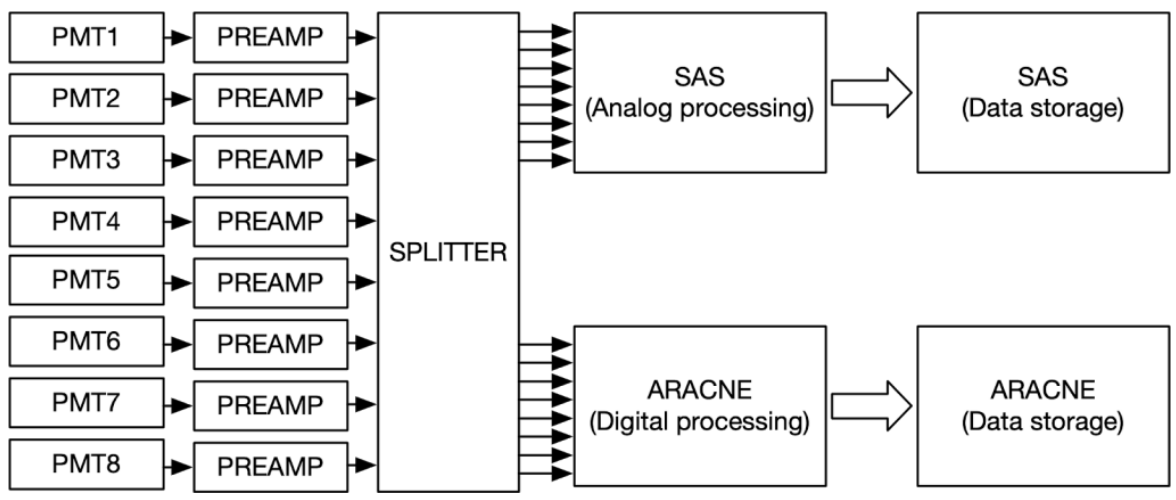

Figure 3. MITO block diagram. The same analogue signals detected by PMTs arrive to both process modules (analogue and digital). In this way, both results can be compared.

The telescope has been equipped with two data acquisition modules that carry out similar functions using different technologies, SAS and ARACNE. Thus, the eight analog signals from the PMTs are first amplified and then fed to both systems in parallel by means of an analog splitter system (Figure 3), so that results from both systems can be compared and validated against each other.

The SAS module performs analog processing on the pulses coming from every PMT, discriminating pulses above and below certain threshold levels, which are per-channel hardware adjustable. The module has four coincidence detectors which can be individually configured to detect a coincidence between any of the eight PMT inputs. The output pulses generated by the four coincidence detectors are counted and registered by means of a low cost Beaglebone Black single-board computer (SBC). At present, we are still developing the pulse height analyzer in this analogue unit.

On the other hand, the ARACNE module (picture in figure 5) works by doing all the discrimination, pulse detection and pulse height analysis digitally. After a simple analog conditioning stage, all 8 signals from the PMTs are sampled in parallel using a high-speed and high-resolution ADC, and a FPGA device monitors all channels simultaneously to detect a pulse on any channel (trigger level and trigger channel(s), among other things, are software configurable). When a pulse is detected, a window of samples of all eight channels, before and after the pulse, is captured and transferred to a SBC (also Beaglebone Black), where coincidence and height of pulses on all channels is determined and stored to determine points of impact on each scintillator as well as muon trajectories. By operating this way, the ARACNE 
module allows pulse counting as well as pulse height (or even shape) analysis, and other kinds of digital processing on the FPGA deemed necessary could be implemented.

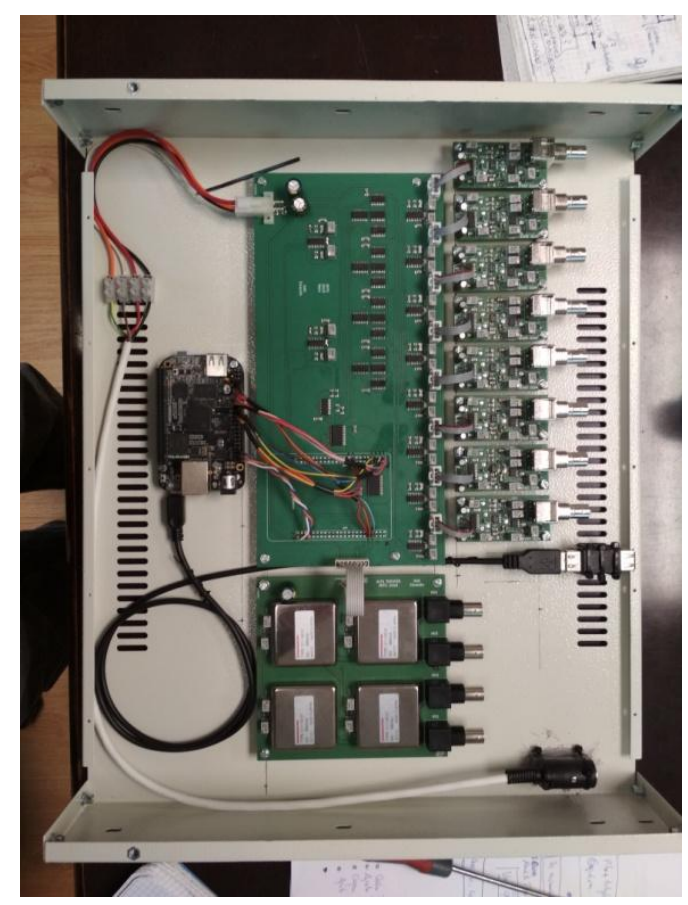

Figure 4. SAS module with the High Voltage module.

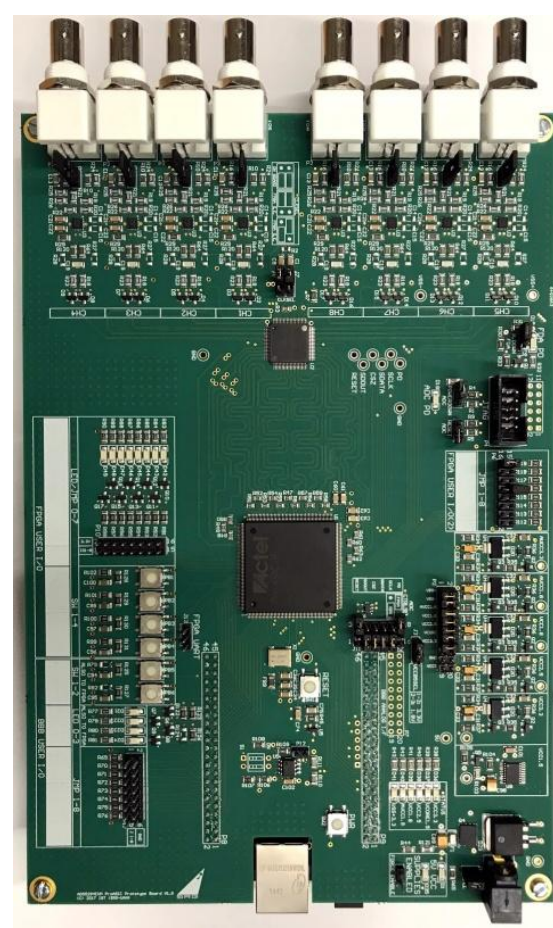

Figure 5. ARACNE module.

\subsection{MITO set-up}

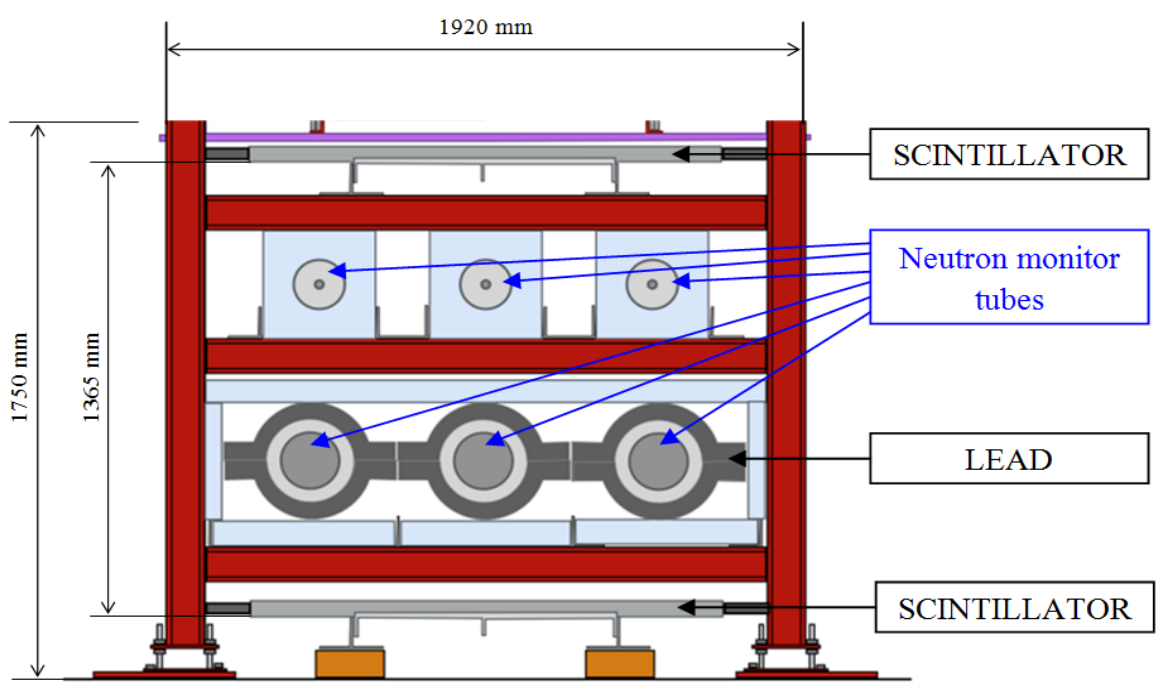

Figure 6. Layout of the final setup. MITO components (scintillators and lead) are integrated in the ORCA assembly.

The two devices conforming the telescope are placed at the top and bottom of a metallic structure, as part of the ORCA project. Between them, the structure also contains the NEMO 
neutron monitor [5][6], part of which is built using a $10 \mathrm{~cm}$ lead layer that consequently stands between the MITO devices (figure 6).

Electronics for data acquisition, storage, atmospheric data, time synchronization and communications were placed into a 19" rack cabinet near the detectors, and the whole setup was accommodated into a 20 feet standard marine container which traveled across the Atlantic Ocean from Spain to Antarctica. The container was placed on the deck of the vessel with its longitudinal axis perpendicular to the longitudinal axis of the ship. In this way, the reference axis (PMT1-PMT3) of the telescope was at 315 degrees (clockwise) with respect to the navigation heading. Temperature, pressure and GPS vessel position were registered during the trip.

\section{Preliminary Data}

MITO performed a latitudinal survey from Vigo (Spain) to Juan Carlos I Spanish Antartic Station from November $14^{\text {th }}, 2018$ to January $2^{\text {nd }}, 2019$. During the journey, some adjustments had to be made, therefore, SAS registered data are reliable from November $17^{\text {th }}$ to December $5^{\text {th }}$ and ARACNE data, from November $26^{\text {th }}$ to January $2^{\text {nd }}$. It must be taken into account that solar activity was extremely low in this period.
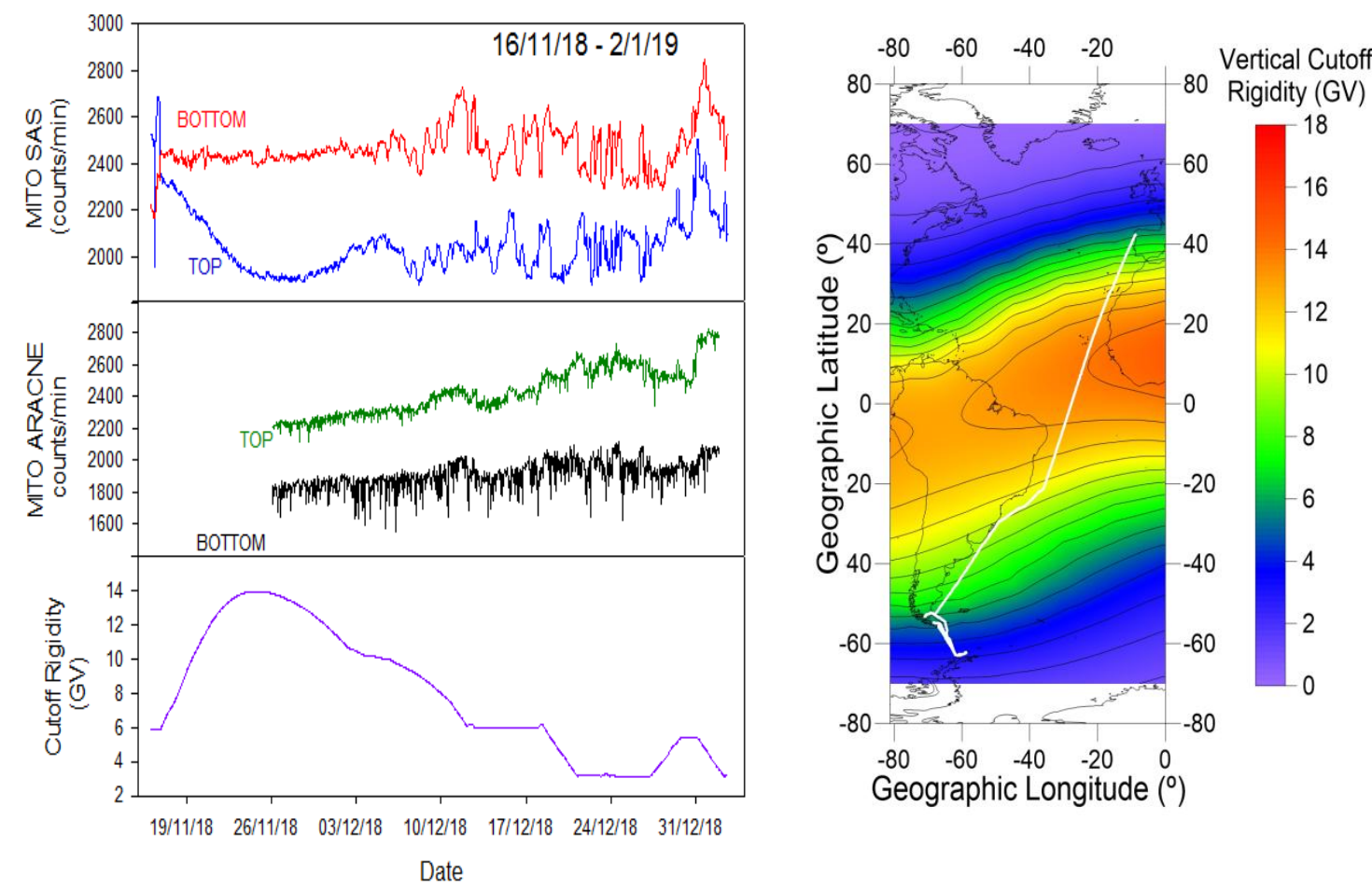

Figure 7. Hourly average data along the survey. The top two panels show raw hourly averaged counting rates registered by SAS and ARACNE from 16/11/2018 to 02/01/2019 (no atmospheric nor temperature correction applied). The bottom panel shows the estimated rigidity cutoff at the vessel location. The right panel shows vertical cutoff rigidities [7] and the vessel route.

Figure 7 shows the muon flux data registered by MITO using both SAS and ARACNE during the survey. The four PMTs on the top scintillator worked in coincidence as well as the 
four PMTs on the bottom, each one in a different coincidence channel. SAS-Top shows a clear decrease, coincident with the maximum cutoff rigidity during the trip. SAS-bottom plot is almost flat, perhaps due to the higher PMT's gain adjustment and the $10 \mathrm{~cm}$ lead layer placed between both scintillators. Both ARACNE's plots show a similar smooth increase as the vessel travels through decreasing cutoff rigidity regions during the trip, although ARACNE-TOP shows a higher slope than ARACNE-bottom.
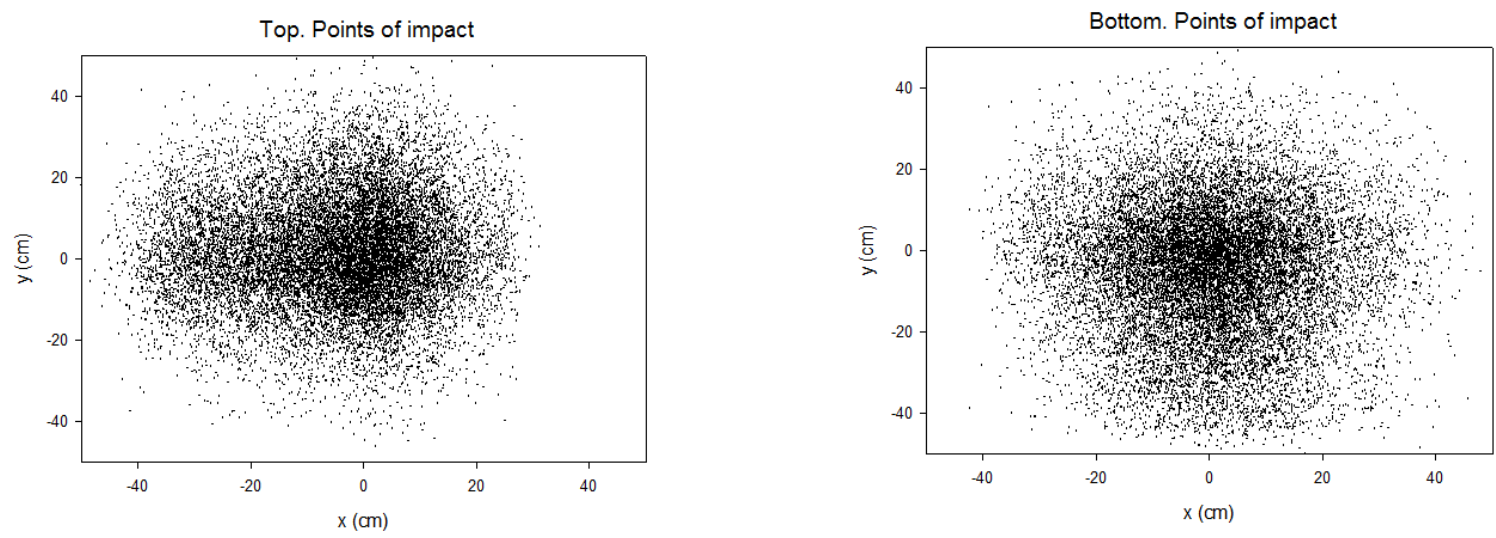

Figure 8. Footprint of impact points in top and bottom scintillators

Apart from counting, MITO is able to analyze and record pulse height from each PMT input, allowing impact point and particle trajectory determination. To do this, an algorithm for point of impact calculation on each scintillator was previously devised and tested by using Monte Carlo simulations. After working out the impact coordinates at each scintillator, the trajectory of the particle can be easily determined.
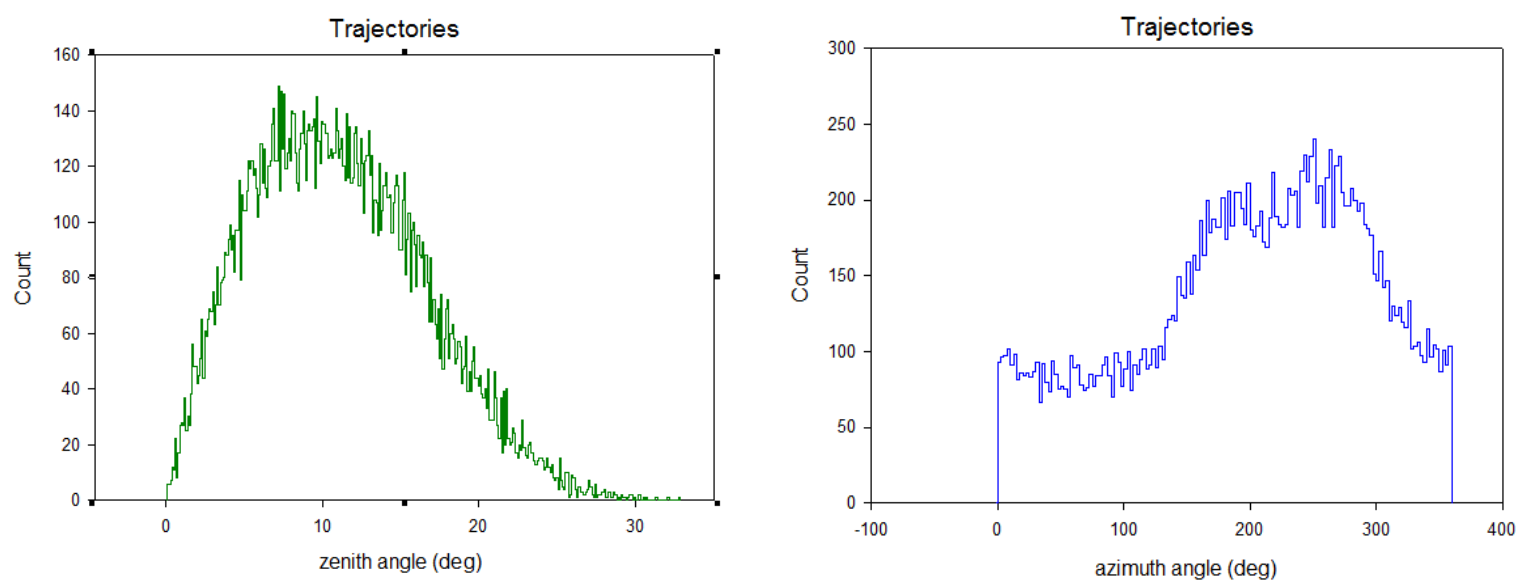

Figure 9: Histograms of zenith and azimuth angle of 20000 trajectories registered on November $30^{\text {th }}, 2018$.

Twenty thousand samples with the 8 PMTs working in coincidence were analyzed from data recorded on November $28^{\text {th }}$, 2018 (8:00-9:00 AM) in order to determine the impact point coordinates on both scintillators, and zenith and azimuth angles of trajectories. Previously, each channel gain was software adjusted so as to equalize their response and, thus, avoid deviations 
in the impact point calculation. Figure 8 shows the inferred preliminary distribution of impact points on both scintillators. As expected, most of impacts are in the central area of the scintillators, due to the geometric factor and to the angular distribution of muon flux at sea level [6].

Calculation of azimuth and zenith angles are shown as an histogram in Figure 9. There is a predominance of incident particles between 289 and 119 degrees with respect to true north, after taking into account the vessel heading $\left(194^{\circ}\right)$ and the MITO orientation with respect to the vessel, as explained above.

\section{Conclusion}

A new muon telescope composed of two stacked scintillators and eight PMTs, able to provide muon count rates and incident directions has been developed and implemented. We present in this poster its design, construction and application, and preliminary results generated from data gathered during the latitudinal survey from Spain to Antarctica.

Although the point of impact calculation algorithms and some electronics have still to be improved and adjusted, preliminary results seem to be reasonably in line with expectations.

\section{References}

[1] S. Cecchini and M. Sioli, Cosmic ray muon physics, Non-accelerator particle physics 201-217, 1998, arXiv:hep-ex/0002052v1

[2] F. Jansen et al, Muon Detectors - the real-time, ground based forecast of geomagnetic storms in Europe, ESA Space Weather Workshop: Looking towards a European Space Weather Programme, 2001, ESA WPP-144W.A. Mozart, Don Giovanni, in proceedings of Mock conference PoS(MC2000)002.

[3] Y. Muraki et al, A data taking system with low power consumption for cosmic ray research, Nuclear Instruments and Methods in Physics Research Section A, Volume 30, Issue 3, 367-368, 2004.

[4] J.J. Blanco, O. García Población, J.I. García Tejedor, J. Medina, M. Prieto, A. López Comazzi, S. Ayuso, R. Gómez-Herrero, C. T. Steigies, A new neutrón monitor at the JuanCarlos I Spanish Antarctic Station (Livingston Island-Antartic Peninsula). PoS(ICRC2019)1059. In P. Desiati, T. Gaisser, and A Karle, editors, 36th International Cosmic Ray Conference ICRC2019), volume 1 of Proceedings of Science, July 2019.

[5] J.J. Blanco, O. García Población, J. I. García Tejedor, C. T. Steigies, J. Medina, M. Prieto, A. LópezComazzi, S. Ayuso, R. Gómez-Herrero, J. A. Garzón, D. García-Castro, P. Cabanelas, A. GomisMoreno, V. Villasante-Marcos, B. Heber, A. Morozova, G. Kornakov, T. Kurtukian, A. Blanco, L. Lopes, J.P. Saravia, H. Kruger, Du T. Strauss, and V. G. Yanke. ORCA 2018 latitudinal survey. PoS(ICRC2019)1060. In P. Desiati, T. Gaisser, and A Karle, editors, 36th International Cosmic Ray Conference (ICRC2019), volume 1 of Proceedings of Science, July 2019.

[6] S. Cecchini and M. Spurio, Atmospheric muons: experimental aspects, Geoscientific Instrumentation, Methods and Data Systems Discussions, Volume 2, Issue 2, 2012, pp.603-641. arXiv:1208.1171v1

[7] D. F. Smart and M. A. Shea. Galactic Cosmic Radiation and Solar Energetic Particles, volume 27, chapter 6, pages 6.1-6.29. Adolph S. Jursa, AIR FORCE GEOPHYSICS LABORATORY, Apr 1988. 